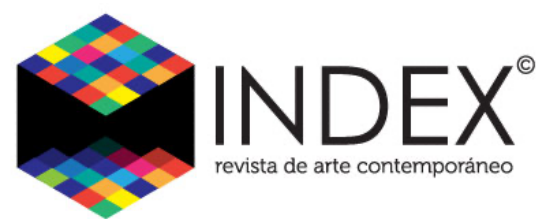

No. 10. DIC 2020 .

Dossier

DOI: $10.26807 /$ cav.vi10.388

\title{
EXPLORANDO LOS DEBATES DE LA INVESTIGACIÓN EN EL CAMPO DEL ARTE
} EXPLORING RESEARCH DEBATES IN THE FIELD OF ART

Lucía Wood

\section{Resumen}

En el presente trabajo me interesa analizar desde una perspectiva metodológica la investigación en el campo del arte. Si consideramos la práctica investigativa no sólo como las acciones puestas en juego en la producción de conocimiento, sino en su complejidad, atravesadas estas acciones a su vez por condicionantes sociales, históricos, culturales, políticos, institucionales, epistemológicos, etc. (Ynoub, 2014); este análisis metodológico no busca proponer criterios clasificatorios y prescripciones sobre cómo debería ser una investigación en este caso en el campo del arte-, sino reflexionar sobre la práctica, problematizarla. Esto implica, entre otras cosas, poder analizar e historizar el debate de la investigación en el campo del arte, contextualizarlo institucional y socialmente, así como considerar los posicionamientos ontológicos y epistemológicos implícitos, los supuestos sobre el arte, el conocimiento, la ciencia, y sus modos de validación.

Palabras clave: ciencia, arte, investigación, conocimiento, práctica

\section{Abstract}

I am interested in researching the art field from a methodological perspective.

Investigative practice may be conceived, both as the actions taking place on knowledge production, as well from its complexity: these actions are influenced by social, historical, cultural, political, institutional, epistemological conditions, etc. (Ynoub, 2014). This methodological analysis proposes a classification criteria or prescriptions about how research may be conducted, in this case, in the field of art. 
Meanwhile, aims to show the practice to problematize it. This implies several aspects. Among them, to analyze the historicizing of the research debate in the field of art, to contextualize it both institutionally and socially and finally, to consider the implicit ontological and epistemological positions, the assumptions about art, knowledge, science, as their validation modes.

Keywords: science, art, research, knowledge, practice

\section{Biografía de la autora}

Lucía Wood (Río Negro, Argentina en 1979; hoy residente en Buenos Aires), Facultad de ArtesUniversidad Nacional de La Plata; Código ORCID 0000-0003-1160-6326. Doctoranda en Artes por la FdA-UNLP; Docente en grado y posgrado, e investigadora en el área de Metodología de la Investigación. Actualmente parte del proyecto I+D "El giro performativo en las artes visuales. A propósito de cuerpos, espacios y objetos puestos en acto”, del Instituto de Investigación en Producción y Enseñanza del Arte Argentino y Latinoamericano, FdA-UNLP, Argentina.

Código de identificación ORCID: 0000-0003-1160-6326

Ya desde hace unos años en el ámbito académico internacional se instaló el debate sobre el estatuto de la investigación en el campo de las artes, solidario de la inclusión de las carreras artísticas en la universidad. Es así como comienza a reflexionarse sobre las particularidades de la currícula, los criterios de evaluación, así como de acreditación y titulación, en los diferentes niveles de formación (grado y posgrado - maestría y doctorado-). 
¿Qué implica la formación en artes en el marco de la universidad? ¿Cómo validar la formación? ¿Pueden implementarse los mismos criterios que en otras carreras universitarias? ¿Es la investigación científica el único criterio posible para acreditar la titulación? ¿Qué se entiende por ciencia, por investigación científica? ¿Qué implica investigar en el campo del arte?

Los desarrollos de este debate que se inicia se despliegan fundamentalmente en tres ejes: la cuestión ontológica (la pregunta sobre el objeto); la cuestión epistemológica (la pregunta sobre el conocimiento), ${ }^{1}$ y la cuestión metodológica (la pregunta sobre los procedimientos) (Borgdorff, 2006; Fajardo-González, s/d). ${ }^{2}$ Predominando el abordaje de las dimensiones ontológica y epistemológica sobre la cuestión, quedando en general limitado a casos particulares los aportes desde la dimensión metodológica.

Este debate surge inicialmente en países europeos y EE. UU., tomando un fuerte impulso hacia fines de la década del 90 con la reforma educativa que inaugura el plan de Bologna (Borgdorff, 2006; López Cano \& San Cristóbal, 2014). ${ }^{3}$ En Latinoamérica la repercusión es

\footnotetext{
${ }^{1}$ A nivel nacional (Argentina) e internacional, encontramos varios/as autores/as interesados/as en profundizar los aspectos ontológicos y epistemológicos, ya sea desde la reconstrucción histórica del abordaje y concepciones de arte y ciencia en los diferentes campos del saber, en particular desde la filosofía, como desde el análisis de los modos de construcción del conocimiento. Varios de los antecedentes destacan así los aportes de Nelson Goodman, quien cuestiona la idea de un único "mundo real", para empezar a pensar en múltiples maneras de hacer mundos, donde la ciencia y arte son maneras diferentes de aludir y contar el mundo. Siguiendo esta línea, se ubica como significativo para considerar en el abordaje actual de la investigación en el campo del arte, el abandono de la concepción del arte como trascendente y verdadero, para reconocer hay algo que permanece incognoscible allí (podríamos pensarlo tanto en la obra como en el proceso de creación), que no por ello es obstáculo a su búsqueda. Solidario esto de reconocer la heterogeneidad en el arte. Para más desarrollo de los antecedentes y algunas de las posturas, pueden consultarse: Wood, 2017; García y Belén, 2015; López Cano y San Cristóbal, 2014.

2 Fajardo-González (s/f) ahonda en el análisis de los antecedentes, fundamentalmente brasileros y latinoamericanos, sobre la investigación en el campo del arte en su dimensión metodológica, con el intento de rescatar propuestas que incluyan, analicen y validen la investigación en artes como modalidad posible en el ámbito universitario. En el ámbito argentino encontramos desarrollos interesantes respecto de los entrecruzamientos entre arte y ciencia en el arte argentino contemporáneo.

${ }^{3}$ El Plan Bologna es el nombre que recibe el proceso iniciado a partir de la Declaración de Bologna, acuerdo que en 1999 firmaron los ministros de Educación de diversos países de Europa (tanto de la Unión Europea como de otros países como Rusia o Turquía), en la ciudad italiana de Bologna. Se trataba de una declaración conjunta que dio inicio a un proceso de convergencia que tenía como objetivo facilitar el intercambio de titulados y adaptar el contenido de los estudios universitarios a las demandas sociales, mejorando su calidad y competitividad a través de una mayor transparencia y un aprendizaje basado en el estudiante cuantificado a través de los créditos ECTS. Para muchos sectores de la sociedad, el Proceso de Bologna va más allá del acuerdo firmado, comprendiendo aspectos relativos a toda la reforma universitaria que se consideran más importantes, especialmente aquellos referidos a la financiación de la universidad pública. Boaventura de Sousa Santos (2018) despliega su crítica, y lo ubica como el intento de mercantilizar la universidad europea, a partir del supuesto que la universidad no sólo
} 
posterior, ya que también es más reciente la incorporación de acuerdos internacionales en la acreditación de la formación en la universidad; así como también se ubica un predominio de modalidades tradicionales de acreditación (López Cano y San Cristóbal (2014) lo plantean fundamentalmente en el caso de las carreras de música).

Es Borgdorff, filósofo y teórico de la música, quien en 2006 en Amsterdam expande el debate sobre la investigación en el campo del arte, diferenciándola en primera instancia de la práctica artística en sí pero, por otra parte, diferenciando en el mismo campo de la investigación, la que denomina la "práctica artística-como-investigación” de la investigación académica (homologada a la que ubica como "científica”). Abre así la pregunta por las diferencias entre estas, bajo el supuesto de que deben conllevar particularidades en lo que hace a la naturaleza del objeto de la práctica artística y de la investigación (perspectiva ontológica); respecto del tipo de conocimiento y comprensión que abarcan (epistemológico); y en los métodos de producción y validación (metodológico).

Es así como algunos autores proponen, a partir de reflexionar sobre la investigación en este campo, la distinción entre la investigación sobre arte, para el arte, y en arte (Borgdorff, 2006; Fajardo-González, s/d). Ubicando a esta última como aquella que podría articular la creación original del artista a través de una obra, así como la reflexión sobre su proceso creativo.

Definen la investigación sobre las artes, como aquella que considera como objeto de estudio la práctica artística entendida ampliamente y en tanto se busca arribar a conclusiones válidas sobre la misma constituyéndose desde una distancia teórica, en la que el objeto de investigación permanece intacto bajo la mirada analítica del investigador/a, desde la que denominan como una "perspectiva interpretativa”. La investigación para las artes es aquella donde “...el arte no es tanto el objeto de investigación, sino su objetivo” (Borgdorff, 2006, p. 8),

tiene que capacitar para el mercado, sino que ella misma tiene que funcionar como el mercado, bajo sus mismas lógicas.

${ }^{4}$ Borgdorff articula en el texto lecturas y presentaciones sobre investigación en las artes a partir de diferentes encuentros académicos durante el 2004 y 2005. Retoma y repiensa una clasificación propuesta con anterioridad por Christopher Frayling en 1993 en el Reino Unido, quien distinguía entre investigación dentro del arte, investigación para el arte e investigación a través del arte (Cabana Bezpalov, 2020). 
aportando descubrimientos, herramientas y técnicas al servicio de la práctica artística, desde una “perspectiva instrumental”. La investigación en las artes “...es el más controvertido de los tres tipos ideales de investigación” (p. 9), no considera distancia entre el investigador/a y la práctica artística, ya que ésta es considerada parte esencial tanto del proceso de investigación como de los resultados de la investigación. Es la que se ubica como "perspectiva de la acción o inmanente". Quien investiga, es el/la artista mismo/a. Hay un intento de articulación de parte del conocimiento expresado a través del proceso creativo y el objeto artístico mismo, donde la práctica artística se entrelaza con la práctica investigativa. ${ }^{5}$

Sonia Vicente ${ }^{6}$ (2006), desde el ámbito académico argentino, parte del análisis de la investigación en el campo del arte de la mano de su inclusión en la universidad y reconstruye históricamente los diferentes tipos de formación e instituciones vinculadas a la misma en el campo artístico. Desde esta perspectiva discrimina las que ubica como posiciones diferentes en lo que hace a la investigación en el campo: 1) quienes ubican a la investigación artística como investigación científica, tomando al arte como objeto de estudio (cercana a lo que Borgdorff ubica como investigación sobre el arte); 2) otra posición que homologa investigación y producción artística (aunque se considera que el arte investiga pero de un modo diferente a la ciencia); y 3) una última posición, a la que adhiere la autora, que considera que la investigación artística presenta una especificidad propia. Desde esta última postura se plantea que el propósito de la investigación artística es producir conocimiento sobre el arte y desde el arte, explicitándolo verbalmente (aunque se ubica que la resultante no sería una construcción teórica sino la explicación de una poética o resolución de problemas artísticos) y haciéndolo circular en ámbitos oficiales académicos y de investigación.

\footnotetext{
${ }^{5}$ Hay que considerar a su vez el marco institucional en cuanto a la formación e investigación en artes en el que se inscribe la posición del autor, Países Bajos. Para mayor detalle, puede verse una entrevista realizada a Bogdorff; en un artículo de Cabana Bezpalov (2020) "El paradigma de la investigación en artes. ¿debate o realidad?".

${ }^{6}$ Sonia Vicente, Profesora de Filosofía y Magíster en Arte Latinoamericano, docente investigadora, profesora titular de las cátedras Filosofía del Arte y Seminario de Licenciatura en la Facultad de Artes y Diseño (UNCuyo, Argentina).
} 
Otros/as autores aportan nuevas lecturas de esta clasificación. Desde una búsqueda similar de la especificidad, Morales López (2009), desde Colombia, diferencia las investigaciones científicas en el campo del arte según sus fines, distinguiendo fines científicos, pedagógicos, y artísticos.

1) con fines científicos, donde el propósito es ampliar el conocimiento del arte y sus problemáticas. Ubicándola como característica de las tesis de posgrado, y las investigaciones científicas. Dentro de este grupo diferencia a su vez las investigaciones en función de los ejes de abordaje de las problemáticas: A) eje en la teorización alrededor del arte; B) eje en la obra artística, apoyándose en los saberes aportados por disciplinas afines, propio de las que ubica como investigaciones hermenéuticas en general; y C) el arte como medio para arribar a otras comprensiones de un fenómeno o de los seres humanos en general.

2) con fines pedagógicos, donde el propósito de la investigación reside en incorporar ciertas prácticas profesionales o socioculturales a la formación de estudiantes.

3) con fines artísticos, caracterizadas por tener como propósito la ampliación del conocimiento técnico-artístico, cultural u otro, necesario para la concreción de un proyecto artístico dado; siendo el resultado esperado de la investigación la producción de obra de arte.

La investigación con fines científicos, podríamos pensarla ligada a la propuesta de Borgdorff de las investigaciones sobre y para el arte. Y la que se orienta por fines artísticos, ligada a la investigación en arte, donde hay un proceso creativo puesto en juego. Ahora bien, ¿qué se entiende por "científico"? Y aquí nos encontramos con la dificultad de encontrar una única respuesta, ya que no hay una concepción unívoca de la ciencia, sino diversidad de definiciones y versiones de ésta. Por lo que la clasificación entre científico/no científico, dependerá de qué se entienda por ciencia.

Desde Argentina, y vinculada a la tercera posición que plantea Morales López, -que representaría la singularidad de la investigación en el campo del arte-, Silvia García y Paola Belén (2015) amplían su análisis y propuesta, centrándose en las posibilidades y características del 
abordaje y resultados esperados con dicho proceso investigativo. ${ }^{7}$ En ese sentido, suponen que, en tanto producción de conocimiento vinculado a lo académico, además de la producción de obra, el/la investigador/a debe centrarse también en la producción de discursos vinculados a dicha obra, que den cuenta de los objetivos, su impacto social, marcos conceptuales que generaron dicha producción, así como su poética. Abriendo esta posibilidad en los que llaman "proyectos de investigación-producción artística". Validando cada uno, obra y discurso, en sus circuitos correspondientes (museos, galerías, etc., unas, y publicaciones en revistas, ponencias en congresos, entre otros). De esta manera consideran podría pensarse una producción en los márgenes de la ciencia instituida.

Las clasificaciones que analizamos son interesantes para abrir el debate y ponernos a pensar, pero en éstas las fronteras son difusas, siendo difícil establecer límites precisos entre un criterio clasificatorio y otro. Donde a su vez es importante considerarlas en tanto construcciones clasificatorias que son resultado del análisis de prácticas concretas que se desarrollan en marcos institucionales, formativos, con posicionamientos epistemológicos y ontológicos subyacentes, muchas veces muy diversos. ${ }^{8}$

Traigo algunos ejemplos para pensar los límites borrosos de las clasificaciones. Sonidos de América es un proyecto artístico y de investigación de un equipo de la Universidad de Villa María, Córdoba (Argentina), donde se articulan la indagación sobre los sonidos de los pueblos originarios de Latinoamérica, con la construcción de las réplicas de instrumentos, y su interpretación y divulgación en conciertos. ${ }^{9}$ Podríamos pensar que en este proyecto se

\footnotetext{
${ }^{7}$ Silvia García es Licenciada en Artes Plásticas, docente e investigadora en Estética; Secretaria de Ciencia y Técnica de la FdA-UNLP, Argentina. Paola Belén es Prof. y Lic. en Historia de las artes visuales (FdA-UNLP). Especialista en Epistemología e Historia de las Ciencias (UNTREF); docente e investigadora en Estética y Epistemología de las artes. Jefa del Departamento de Estudios Históricos y Sociales (FdA-UNLP).

${ }^{8}$ Los límites difusos en su clasificación, así como la importancia de considerar las particularidades de los marcos institucionales de las universidades/institutos, así como los condicionamientos a partir de las políticas educativas y de investigación en cada país, son aspectos que también ubica Borgdorff en la entrevista de 2019.

${ }^{9}$ Sonidos de América es una investigación en la que se unen la música, la arqueología y la cerámica. Se busca estudiar la sonoridad perdida de la América pre-hispánica, a partir del análisis de los utensilios utilizados por los pueblos y sus piezas cerámicas, algunos considerados posibles instrumentos musicales. Definen su rama de trabajo como radioarqueomusicología, ya que las piezas cerámicas son sometidas a rayos X para poder descubrir cómo era posible que emitieran sonidos, algunas por efecto del aire y otras por acción del agua. Además del estudio
} 
entrecruzan aspectos característicos de las que ubican como investigación sobre, para y en arte (Borgdorff, 2006), así como con fines científicos y artísticos (Morales López, 2009). Otro ejemplo es el de Elizabeth Thompson, artista y docente de la Escuela de Artes Plásticas de la Universidad de Costa Rica (Zuñiga Salas, 2015), quien lleva adelante su investigación con el objetivo de estudiar tintes extraídos de plantas propias del entorno costarricense para aplicarlos a la pulpa de celulosa, con fines artísticos y de conservación. ${ }^{10}$ En este caso encontramos aspectos relativos a las denominadas investigación para y en arte (Borgdorff, 2006), así como con fines artísticos, científicos y pedagógicos (Morales López, 2009). Vemos con estos ejemplos que las clasificaciones son porosas, se entrelazan en la práctica, y ésta las excede, invitándonos a complejizar la mirada. Nos muestran que una misma investigación puede pensarse analizando e interpretando cierta producción/proceso artístico (sobre arte), que a su vez aporte instrumentalmente al campo (para el arte), donde asimismo la práctica artística sea un eje central en la investigación (investigación en arte, o con fines artísticos), y compartiendo a su vez espacios de divulgación y metódicas propias de ciertas tradiciones académicas y científicas. Nos intentamos por tanto correr de las pretensiones clasificatorias, para encontrar otras aristas que permitan abordar la investigación en el campo del arte.

Es interesante notar que es un debate aún abierto, no tanto por las diferencias de criterios de las clasificaciones propuestas, sino sobre todo porque nos encontramos en un tiempo en que la misma práctica se está pensando y construyendo a sí misma. Desde la metodología, nos pensamos

sonoro, la investigación implica la construcción de piezas, "cuando empecé a construir empecé a tener otra forma de tocar y a descubrir muchas más cosas (entrevista a Esteban Valdivia, 2013)". La otra parte del proyecto la consideran fundamentalmente creativa, "de performance artística", dada por la difusión e interpretación de las piezas y objetos en diferentes espacios culturales y académicos, acompañada por la presentación de las producciones audiovisuales y fotográficas del proyecto. Con esto se busca a su vez que este conocimiento esté en contacto con la gente, que puedan probar los instrumentos y vivenciar la sonoridad (Ferreras, 2013; videos Sonidos de América, 2011, 2012, 2017).

10 Es una investigación que lleva más de 10 años, en cuyo proceso han participado colegas de la investigadora, así como estudiantes. El estudio de los tintes naturales se articula con la práctica artística - pintura, arte textil, escultura-, donde se pone en acto lo analizado. Entre los resultados se han logrado investigar fuentes de colorantes naturales como el achiote, el azul de mata, el aguacate, el eucalipto, la cúrcuma, entre otros; en papeles hechos a partir de pulpa papelera de cabuya, itabo, escobilla y morera. Según la artista “...sus procesos de investigación están permeados por la herencia del método científico, en una especie de hibridez con sus procesos creativos" (Zuñiga Salas, 2015, p.60). 
como campo que analiza retrospectivamente las prácticas investigativas (Ynoub, 2014), y en este caso, nos encontramos aún en un tiempo instituyente de dicha praxis en el campo artístico.

\section{Reflexiones sobre las concepciones de ciencia, arte, y el lugar de las instituciones}

García y Belén (2015) abren en su análisis un nuevo interrogante: ¿̨resulta necesario que la investigación en artes sea científica? En este punto la pregunta debe girar entorno de los modos de validación del conocimiento, entendiendo que el científico no es el único tipo de conocimiento posible, pero no por esto desconociendo el lugar hegemónico que se le ha dado en la universidad y la sociedad.

Vemos que no hay un único modo de pensar la práctica investigativa en el campo del arte. Así como tampoco hay una concepción unívoca sobre la ciencia y la manera de comprender la práctica investigativa científica. Ésta, propongo pensarla como una práctica social, atravesada a su vez por aspectos subjetivos, que busca reflexionar y problematizar las teorías y prácticas de un campo, donde también se pone en juego un proceso creativo, de producción de nuevos conocimientos, que va más allá del resultado concreto de la investigación. Y que, como práctica, se tensiona entre lo instituido y lo instituyente, supone su inscripción en ciertas tradiciones y paradigmas que la orientan y validan, y a su vez, debe permanecer abierta a la posibilidad de nuevos modos.

No hay temas científicos o no, sino lo que daría el carácter de "científico" al conocimiento son los criterios en los que se sustenta su producción y validación. En tanto este conocimiento se tensiona entre su alcance conceptual, su coherencia teórica, y su validación empírica; mediación que posibilita la praxis (Samaja, 1993; Ynoub, 2014). ${ }^{11}$ Samaja ubica al descubrimiento y la validación, como los dos modos que hacen al método general de la ciencia, diferenciándolo de las metódicas o técnicas particulares que cada disciplina o campo fue

\footnotetext{
${ }^{11}$ Juan Samaja (1941 -2007), de Argentina, prof. Filosofía, Lic. Sociología, Doctor en Ciencias, epistemólogo reconocido por sus contribuciones en las áreas de Filosofía de la Ciencia, Metodología de la Investigación y Semiótica. Roxana Ynoub es argentina, Doctora en Psicología. Se ha especializado en Metodología de la Investigación, Semiótica e investigación hermenéutica, Psicogénesis, realizando aportes a la Metodología de la Investigación desde una perspectiva crítica.
} 
consolidando en el marco de sus propias tradiciones. ${ }^{12}$ La práctica artística, si bien también supone una inscripción en ciertos modelos y trayectorias, no tiene estas mismas exigencias de validación. Y esto nos trae a reflexionar sobre el marco institucional académico universitario, como condicionante a su vez de la formación, la práctica y la producción de conocimiento.

Oscar Cornago ${ }^{13}$ (2015) en este punto ubica una contradicción en la búsqueda de homologar a ciertas metodologías (que la legitimen como formas de investigación) a las artes, cuando la función de éstas dice, consiste justamente en el cuestionamiento de los formatos autorizados. Invita a revisar qué entendemos por arte y ciencia, y reconsiderar el lugar del conocimiento frente a la sociedad, buscando mayor cercanía, interacción, apertura, y así reconsiderar a su vez el lugar de la teoría frente a la práctica. Para esto analiza el lugar de la formación académica en artes, dando cuenta de la separación entre práctica y teoría, donde la legitimación académica sobre todo recae en el conocimiento teórico. Ubica un escenario cambiante en este aspecto, con la posibilidad de un tipo de investigación específica en artes (con resistencias tanto dentro de los ámbitos de homologación institucional, como del espacio de creación). ${ }^{14}$

En una búsqueda similar, Sánchez Martínez ${ }^{15}$ (2009) plantea que lo específico de la investigación en artes estaría dado por la creatividad, por lo que su eje debe volcarse sobre ésta, sobre el análisis del proceso de creación, para así permitir la posibilidad de no sólo transmitir y formar en el manejo de las técnicas, sino a su vez posibilitar la formación en metodologías

\footnotetext{
${ }^{12}$ La vinculación entre el modo de descubrimiento y el modo de validación ha constituido un eje sustantivo de abordaje en las perspectivas constructivistas o historicistas, en oposición a posiciones positivistas y prescriptivistas sobre la ciencia y la metodología de la investigación. Vemos así que no hay un único modo de entender la ciencia y la práctica investigativa. Así como es importante revisar e incluir aspectos que han quedado por fuera de la sistematización tradicional del conocimiento científico, en cuanto a su proceso productivo, sus modos de trasmisión y circulación, etc.

${ }^{13}$ Óscar Cornago (Madrid, España, 1969) es investigador del Centro de Ciencias Humanas y Sociales del Consejo Superior de Investigaciones Científicas de Madrid. Entre sus ejes de trabajo, se destacan los vinculados a la performatividad, y aquellos orientados a repensar la creación escénica replanteando las relaciones entre las teorías críticas y las prácticas de creación (extraído de: http://archivoartea.uclm.es/; el 05/10/20).

${ }^{14}$ Aquí hay que considerar la diversidad entre los países e instituciones, a raíz de sus políticas educativas, científicas, y reglamentaciones marco de las carreras y sistema investigativo.

${ }^{15}$ José Antonio Sánchez Martínez, es Doctor en Filosofía, investigador y catedrático de Historia del Arte en la Universidad de Castilla-La Mancha, España; autor de numerosos textos críticos sobre artes escénicas, literatura y cine.
} 
creativas. Parte de una concepción del arte ligado primeramente a su condición de "experiencia", más allá de su vinculación con el lenguaje. ${ }^{16}$ Abriendo a pensar que la relación que inaugura la práctica artística es multilateral, no sólo reducida a emisor-receptor, sino incluyendo las instituciones, el contexto social, con referentes del pasado, desde lo efectivo del presente, así como sus lecturas futuras. ${ }^{17}$ La experiencia estética estaría por tanto más allá de los límites de las disciplinas, por lo que se debe buscar acercar la teoría a la realidad cotidiana.

Su propuesta por tanto se orienta a que los/as académicos/as no tratemos de inventar nuevas metodologías que enseñar a los/as artistas, sino que busquemos en el trabajo de quienes han investigado en su creación para encontrar las metodologías a aplicar en la academia. Es la academia la que debería desplazarse al terreno artístico y no a la inversa (Sánchez Martínez, 2016). ${ }^{18}$

Por otra parte, desde la perspectiva decolonial, se nos propone repensar críticamente las condiciones de producción del conocimiento en los países coloniales-periféricos, aportando a revisar nuestras instituciones y prácticas. Siendo el paradigma aún dominante el modelo de racionalidad que preside la ciencia moderna, según Castro-Gómez ${ }^{19}$, se reproduce: “...la universidad como lugar privilegiado de la producción de conocimientos (...) una institución que establece las fronteras entre el conocimiento útil y el inútil, entre la doxa y la episteme, entre el conocimiento legítimo (es decir, el que goza de "validez científica”) y el conocimiento ilegítimo" (2007, p. 81). Anclado esto en lo que denomina la estructura triangular de la colonialidad: la colonialidad del ser (ontológica), la colonialidad del poder y la colonialidad del saber (gnoseológica).

\footnotetext{
${ }^{16}$ Concepción que los/as teóricos/as del arte contemporáneo han profundizado en diferentes aspectos.

${ }^{17}$ Esta concepción del arte rompe con la tradicional mirada moderna, centrando la importancia en el "hacer" por sobre el producto, desplazando el eje de la creación (de un producto=obra) a la práctica (el proceso creativo).

${ }^{18}$ En su defensa de la investigación artística basada en la práctica, Sánchez (2016) introduce el tema a partir de la que plantea como necesidad de revisión de 4 conceptos eje: conocimiento, sujeto, comunicación y contexto; a partir de la inclusión de otras 4 categorías: corporalidad, situación, proceso y compromiso. En la búsqueda de un abordaje que considere la complejidad de la investigación científica así como de la práctica artística, proponiendo a su vez puntos de intersección que abran a un nuevo tipo de práctica académica.

${ }^{19}$ Santiago Castro-Gómez, es filósofo colombiano estudioso de las herencias coloniales, que formó parte del Grupo modernidad/colonialidad.
} 
De allí su propuesta de decolonizar la universidad - decolonizar las instituciones, y con éstas el conocimiento y las propias prácticas.

La institucionalización y burocratización del conocimiento cierran las posibilidades de diálogo con otros saberes, tornándose necesario pensar usos contrahegemónicos de la ciencia, no negándola, sino visibilizando su diversidad, así como la presencia y valor de otras formas de saber.

El pensamiento contemporáneo ha desmitificado las seguridades de la razón moderna, dando cuenta que la razón demostrativa dice algo de la realidad, pero limitada respecto de la complejidad de lo real. La "verdad" se empieza a pensar no sólo como patrimonio de la ciencia, sino que también puede ser develada, por ejemplo, por una obra artística. ${ }^{20}$

En este sentido, no se trataría de la oposición arte versus ciencia, o de ajustar la práctica artística a los criterios de validación de la investigación científica, o a la inversa; sino pensar la posibilidad de coexistencia de múltiples saberes, modos de conocer, y que estos puedan enriquecerse mutuamente y participar de diversas maneras en la práctica. ${ }^{21}$ Poder pensar más allá de las polaridades dicotómicas y los enfrentamientos. Reconociendo a su vez la multiplicidad y diversidad al interior mismo de cada campo, permitiéndonos reconocer "las" artes, "las" investigaciones, las versiones de ciencia.

\footnotetext{
${ }^{20}$ Mónica Caballero (2016) refiere en este punto, al análisis de Heidegger sobre la esencia del arte en relación a la operación de desocultamiento de una "verdad".

${ }^{21}$ De Sousa Santos (2018) recupera la importancia de la universidad como lugar de pensamiento, y por ende como posible espacio de resistencia a la compleja trama de la dominación actual entre colonialismo, capitalismo, y patriarcado. Para posibilitar esta construcción de alternativas, ubica la necesidad de instaurar dos rupturas centrales en y desde la universidad: una ruptura epistemológica, en tanto dar lugar, abrirse, ampliar, diversificar, dialogar y construir junto con otros saberes más allá del conocimiento científico; y una ruptura relativa al lugar de la universidad en lo social, como reproductora de una estructura de exclusión, posibilitando un corrimiento hacia un lugar de encuentro genuino y construcción colectiva del conocimiento con los otros excluidos, en lo que ubica como una universidad "intensa", que integre saberes, y que a su vez articule solidariamente con otros actores e instituciones. La propuesta del autor (2015) de la "ecología de saberes" como superadora de la monocultura de la ciencia moderna, supone el reconocimiento de la pluralidad y heterogeneidad de saberes, fundándose en la idea del conocimiento como inter-conocimiento.
} 
Rita Segato 22 (2017) en este punto diferencia el "dualismo" de los pueblos originarios —donde el uno y el dos son posibilidades de lo múltiple-, del "binarismo" propio de la colonial modernidad, resultante de una episteme de la exclusión, del mundo del Uno, donde todas las formas de otredad con relación a este patrón universal constituyen un problema. La ciencia occidental moderna lo reduce en el binarismo sujeto/objeto. ${ }^{23}$ La propuesta decolonial implica intentar abrir brechas en este territorio totalizado por el esquema binario. Pensar más allá de los opuestos, permitiéndonos aceptar las contradicciones, considerar y valorar la multiplicidad y la diversidad.

La propuesta es la de advertir qué condiciones habilitan y legitiman esos lugares instituidos social y culturalmente, para poder intervenir allí, fracturando este posicionamiento polar. Qué replicamos o ponemos en acto sosteniendo la legitimidad de un modo de conocer por sobre el otro, qué posicionamientos epistémicos reproducimos en nuestras prácticas docentes e investigativas, así como en otras esferas de la vida. Esto permite complejizar la mirada sobre la propia práctica, y pensar que estos posicionamientos son también sociales, políticos, suponen relaciones de poder. Poder advertir este movimiento epistémico, nos permitirá también su apertura en nuestras prácticas. Poder corrernos de la lógica prescriptiva, normativa, para dar lugar y hacer con lo complejo.

\section{Referencias}

Azaretto, C. \& Wood L. \& otros (2016). Investigar en Arte. Libro de Cátedra. EDULP, La Plata, Argentina.

\footnotetext{
${ }^{22}$ Rita Segato, antropóloga, y activista feminista argentina. Docente e investigadora en cuestiones de género en los pueblos originarios y comunidades latinoamericanas, violencia de género, y a las relaciones entre género, racismo y colonialidad.

${ }^{23}$ La preponderancia de polaridades binarias o dicotómicas, sujeto-objeto, teoría-práctica, naturaleza-cultura, individuo-sociedad, mente-cuerpo, civilización-barbarie, etc., que instaura el pensamiento racional moderno, hoy empieza a entrar en crisis con las teorías de la complejidad.
} 
Borgdorff, H. (2006). "El debate sobre la investigación en las artes”. Amsterdam, Holanda. Actas - VI Congreso Internacional Latina de Comunicación Social - VI CILCS Universidad de La Laguna, diciembre 2014.

Caballero M. (2016). Teoría de la práctica artística. Fundamentos para una mirada situada del campo estético y cultural; EDULP, La Plata, Argentina.

Cabana Bezpalov, E. (2020). “El paradigma de la investigación en artes ¿debate o realidad?”. Index, Revista De Arte contemporáneo, (09), 122-128. https://doi.org/10.26807/cav.v0i09.332

Castro-Gómez (2007). "Decolonizar la universidad. La hybris del punto cero y el diálogo de saberes"; en Castro-Gómez, S. \& Grosfoguel, R. (eds.) El giro decolonial. Reflexiones para una diversidad epistémica más allá del capitalismo global; Siglo del Hombre Editores, Bogotá, Colombia.

Cornago Óscar. (2015). Ensayos de teoría escénica sobre teatralidad, público y democracia; Madrid, Abada Editores, PP. 56-63.

De Sousa Santos, B. (2015). Una Epistemología del Sur; Ed. Siglo XXI, Buenos Aires.

De Sousa Santos, B. (2018). Conferencia del 11/06/18 en el marco de la III Conferencia Regional de Educación Superior (CRES) en Córdoba, Argentina. Disponible en: https://www.youtube.com/watch?v=8EGLVBsSH8c

Fajardo- González, R. (s/f). "La investigación en el campo de las Artes Visuales y el ámbito académico universitario", Grupo de Estudios Peirceanos. Disponible en: www.unav.es/gep/InvestigacionArtesFajardo.pdf

Ferreras, A. (2013). "Esteban Valdivia: rastreador de sonidos"; entrevista a Esteban Valdivia, diario La Voz del 05/12/2013. http://www.lavoz.com.ar/ciudad-equis/rastreador-desonidos. 
García S. \& Belén P. (2013). Aportes epistemológicos y metodológicos a la investigación artística.

Fundamentos, conceptos y diseño de proyectos; Editorial Académica Española, Saarbrücken, Alemania.

López Cano R. \& San Cristóbal (2014) El dilema de la investigación artistica; trabajo presentando en Anais do III SIMPOM 2014 - Simpósio Brasileiro de pós-graduandos em Música. Disponible en:

http://www.seer.unirio.br/index.php/simpom/article/viewFile/4482/4055

Morales-López, P. (2009). “Investigar el arte: provocaciones para una reflexión necesaria”. En Paradigmas, [número especial], 11-29; Bogotá, Colombia.

Samaja, J (1993). Epistemología y metodología. Eudeba. Buenos Aires, Argentina.

Sánchez Martínez, J. A. (2016). “In-definiciones. El campo abierto de la investigación en artes”. Artes La Revista, 12(19), 36 - 51. Recuperado a partir de https://revistas.udea.edu.co/index.php/artesudea/article/view/26282

Sánchez Martínez, J. A. (2009) «Dosier “Scanner”. Investigación y experiencia. Metodologías de la investigación creativa en artes escénicas». Estudis escènics: quaderns de l'Institut del Teatre, [en línia], 2009, Núm. 35, p. 327-35, https://www.raco.cat/index.php/EstudisEscenics/article/view/252850 [Consulta: 5-102020].

Segato Rita (2017). La guerra contra las mujeres; Ed. Tinta Limón, Buenos Aires, Argentina.

Vicente S. (2003). “Arte y Ciencia. Reflexiones en torno a sus relaciones”; publicado en la revista Huellas, No3/año2003/pág 85-94, Mendoza, Argentina.

Vicente S. (2006). “Arte y parte. La controvertida cuestión de la investigación artística”; en René Gotthelf (director) La investigación desde sus protagonistas. Senderos y estrategias; Editorial de la UNCuyo; Mendoza, Argentina. 
Wood L. (2017). La investigación en el campo del arte. Aportes metodológicos a la cuestión; material de cátedra de Metodología de la Investigación en Artes (FdA-UNLP), Argentina.

Ynoub, R. (2014). Cuestión de Método. Apuntes para una metodología crítica. Cengage Learning Editores. México DF.

Zuñiga Salas, X. (2015). "La investigación artística en las artes visuales: Estudio de tres casos en la Universidad de Costa Rica”. Disponible en:

http://revistas.ucr.ac.cr/index.php/escena/article/view/21175

Enviado: 2020-10-05

Aceptado: 2020-12-06 